\title{
Clinician Decision Support Initiative to Decrease Outpatient High-Risk Medicine Prescriptions in the Elderly
}

\author{
Corinne Rhodes, MD MPH' (D), Jeffrey Tokazewski, MDㄱ, Kristin Christensen, $\mathrm{MD}^{7}$, \\ Maureen Holman, CRNP ${ }^{7}$, Ami Eimers, MSC ${ }^{2}$, and Maryanne Peifer, $\mathrm{MD}^{7}$ \\ 'University of Pennsylvania Health System, Philadelphia, PA, USA; ${ }^{2}$ TMF Health Quality Institute, Austin, TX, USA.
}

$\mathrm{J}$ Gen Intern Med 35(8):2492-4

DOI: $10.1007 / \mathrm{s} 11606-019-05556-9$

() Society of General Internal Medicine 2019

\section{INTRODUCTION}

Reduction of high-risk medications (HRM) in the elderly (age 65+) was identified as an opportunity for improvement in a clinic-based quality improvement (QI) program at one academic suburban outpatient clinic when assessing electronic clinical quality measures within a Medicare demonstration grant Comprehensive Primary Care Plus (CPC+).

Previous studies have shown HRM in elderly, as defined by the American Geriatrics Society BEERS list in the USA, ${ }^{1}$ lead to increased odds of adverse drug reactions (OR $1.4495 \% \mathrm{CI}$ 1.33-1.56) and hospitalizations (1.27 95\% CL 1.2-1.35). ${ }^{2}$ Strategies to reduce risk of adverse drug events include discontinuing medications and prescribing new medications sparingly. $^{3}$

QI work at initial site supported by our institution's process improvement in action program included a mixed intervention: brief peer education, monthly peer comparison report cards, patient-targeted informational posters, and lowtechnical refill clinical decision support from a single licensed practical nurse. This work targeted the clinic's three most prescribed HRM in elderly (cyclobenzaprine, lorazepam, and zolpidem) and resulted in an initial $20.5 \%$ reduction 3 months after intervention with persistent $10 \%$ reduction from baseline at 6 months' post intervention.

Our primary care service line (PCSL) sought to generalize this successful local QI initiative. The aim was to use central informatics resources to develop and implement a sustainable electronic health record (EHR) clinical decision support tool to provide actionable, evidence-based decision support to decrease unnecessary use of HRM in our elderly population. This aim aligned with major payor programs like measure-based incentive payments and $\mathrm{CPC}+$.

\section{METHODS}

Our large academic PCSL includes $\sim 40$ practices and $~$ 93,000 geriatrics patients with a single EHR and dashboard tracking HRM prescriptions for elderly patients. Decision support provided at the point of care for the most commonly prescribed HRM in elderly in the PCSL from BEERS list: zolpidem, promethazine, diphenhydramine, cyclobenzaprine, and amitriptyline. Best practice advisory (BPA) fires with any prescription and provides a soft stop providing BEERS list alternatives ${ }^{4}$ to HRM in elderly depending on indication of use (Fig. 1(a)). For existing medications, during a refill encounter, new functionality indicates that patients "fail" refill protocol based on age (Fig. 1(b)). The patient-facing posters were distributed to all PCSL practices for use.

We monitored HRM prescription rates via run charts to track the ongoing prescription rates per 1000 elderly patients in a pre/post analyses to track changes in monthly average prescribing rates. Clinics new to our EHR during analysis period had a minimum of 2 months baseline data. We also tracked rate of BPA triggers versus conversion to HRM prescription versus alternatives.

This project was reviewed and determined to qualify as quality improvement by the University of Pennsylvania's Institutional Review Board.

\section{RESULTS}

Run chart (Fig. 2) showed reduction in average monthly rates of elderly patients with HRM prescription per 1000 patients from 20.60 to 19.72 , a relative $4.3 \%$ reduction, a shift in baseline average but not significant with 3-5 consecutive points below prior run chart average.

BPA data showed 1273 individual triggers from April-June 2019 for new HRM in elderly prescriptions. Of these, 163 events showed a prescription within 6 days, showing $13 \%$ conversion to prescription of HRM after BPA.

\section{DISCUSSION}

This low-cost, low-tech non-sustainable successful QI intervention reducing HRM in elderly was successfully implemented as PCSL sustainable intervention using clinical 


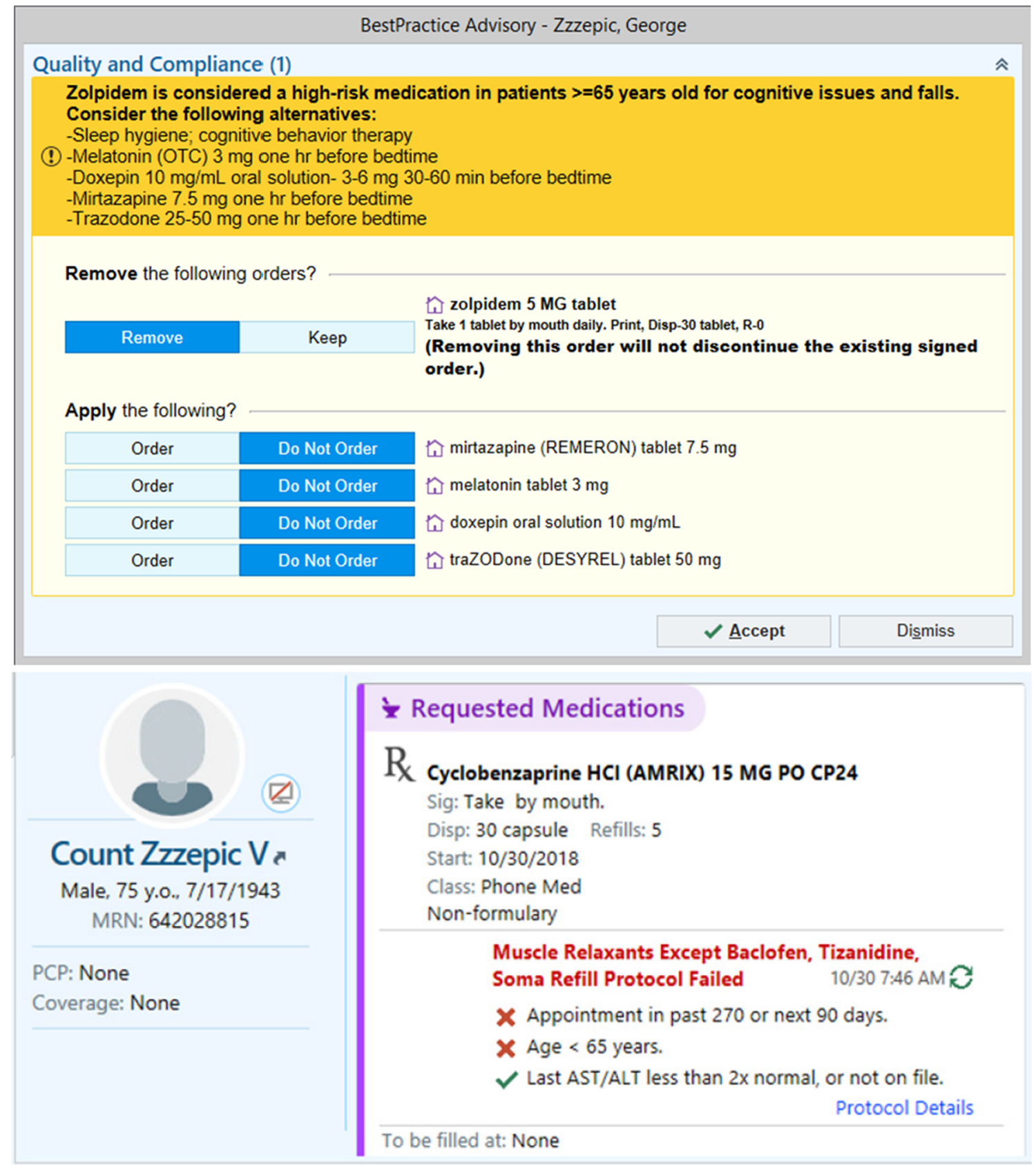

Figure 1 Best practice alert (top 1 (a)) and medication refill (bottom 1 (b)) screen shots.

decision support software as a BPA. Process metric of $13 \%$ conversion of HRM prescription in elderly shows success, although the relative reduction in overall elderly patients with prescription $(4.3 \%)$ was more modest than the initial site reduction $(10 \%)$.

Limitations include inability to assess change of provider behavior with refill-encounter clinical decision support which did not include soft stop nor prescription alternatives. The migration of new clinics to EHR may have contributed to variable baseline data which could affect intervention analyses. We were unable to track adverse drug events from HRM nor track potentially negative unintended consequences from alternative medication prescriptions. 


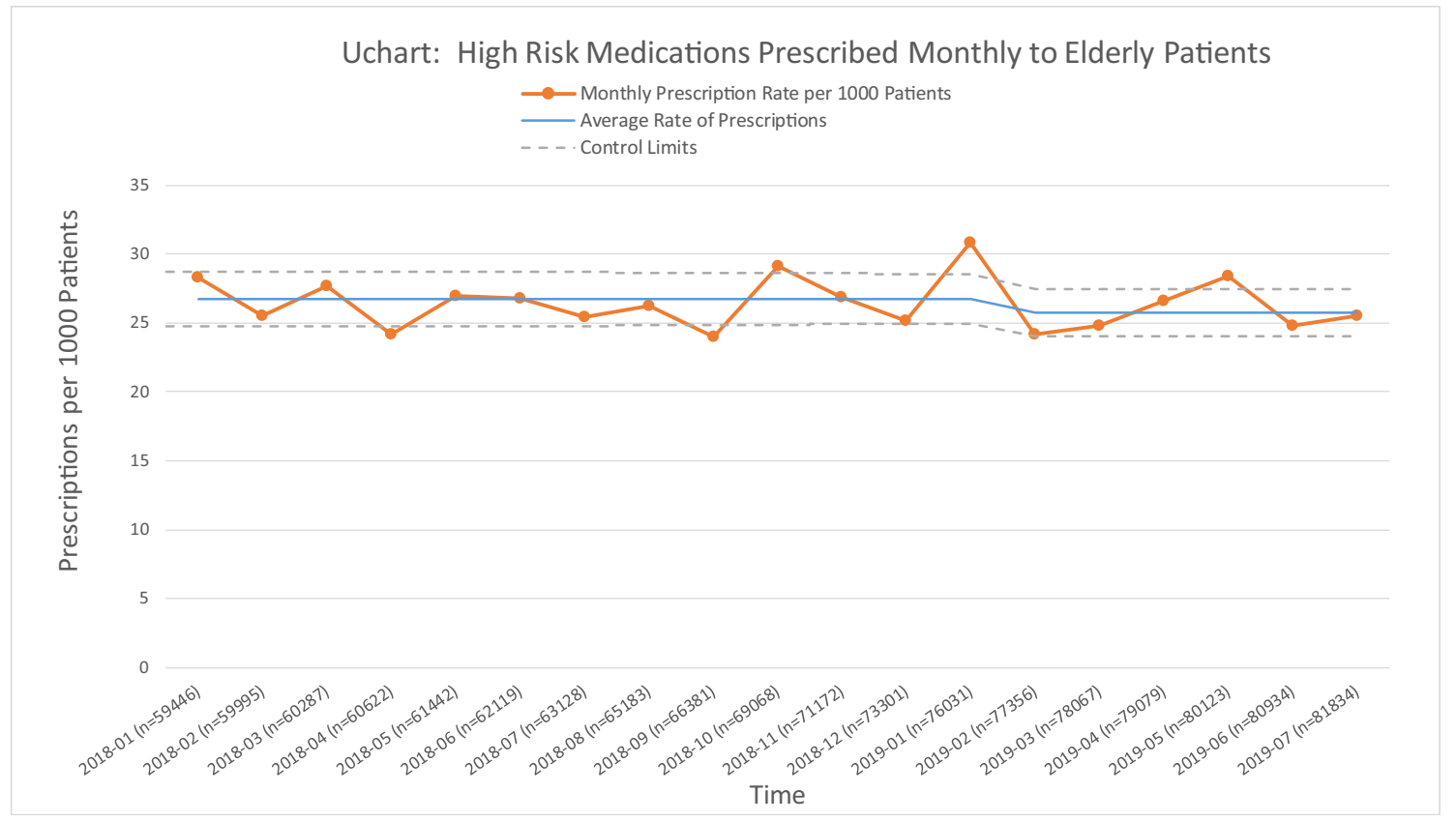

Figure 2 Run U-chart: Tracking number of High-Risk Medications Prescribed monthly to elderly patients

In conclusion, this soft-stop active BPA-reduced prescription of HRM in elderly for new medication orders was a relatively small systems intervention with great reach. Based on this work, our health system is extending this work to deploy BPA in specialty clinics.

Corresponding Author: Corinne Rhodes, MD MPH; University of Pennsylvania Health System, Philadelphia, PA, USA (e-mail: corinne. rhodes@pennmedicine.upenn.edu).

\section{Compliance with Ethical Standards:}

Conflict of Interest: The authors declare that there is no conflict of interest.

\section{REFERENCES}

1. 2019 American Geriatrics Society Beers Criteria $\circledR^{\circledR}$ Update Expert Panel. American Geriatrics Society 2019 Updated AGS Beers Criteria ${ }^{\circledR}$ for
Potentially Inappropriate Medication Use in Older Adults. J Am Geriatr Soc. 2019 ;67(4):674-694

2. Xing XX, Zhu C, Liang HY, Wang K, Chu YQ, Zhao LB, Jiang C, Wang YQ, Yan SY. Associations Between Potentially Inappropriate Medications and Adverse Health Outcomes in the Elderly: A Systematic Review and Metaanalysis. Ann Pharmacother. 2019;53(10):1005-1019. doi: https://doi. org/10.1177/1060028019853069.

3. PRETORIUS, RW, Gataric, G, Swedlund S, and Millier, J. Reducing the Risk of Adverse Drug Events in Older Adults. Am Fam Physician. 2013;87(5):331-336.

4. Healthy Aging: BEERS list and alternatives. https://www.healthinaging. org/medications-older-adults/ Accessed July 15, 2019

Publisher's Note Springer Nature remains neutral with regard to jurisdictional claims in published maps and institutional affiliations. 